\title{
Editorial: Architecture as a Communicative Medium
}

\author{
Anijo Punnen Mathew, Assistant Professor \\ Institute of Design \\ Illinois Institute of Technology
}

Environmental psychology categorizes "physical environment" as "typically neutral," only coming into selfconscious awareness when individuals form stable and enduring representations of it [1]. We see this manifest in the real world when the steeple of a church is able to communicate that it is a place for reflection and religious gathering; the bricks and ivy of Harvard Yard signifies years of scholarly research and education; the solid grey walls of a prison conjures up images of torture and punishment; and the bright colors of a playground indicates play and joyfulness. In our relationship with architecture, we are able to construct an understanding of our environment because we pick up such clues and cues from parts of our environment and then construct a formulation of the whole based on our memories and knowledge. Thus, we are able to communicate with the places we live in and the places we live in are able to act as interfaces for information interchange.

One theory [3] linking linguistics and architecture claims that within design, graphic tokens - shapes, structural forms etc. (like words) are defined in complex logic systems - hierarchy, semblance etc. (like grammar). This constitution of tokens and grammar is what enables us to construct and read into our built environment. Snodgrass and Coyne [5], on the other hand, claim that this model is an instrumental view of language which sees design as a tool, as something external to the subject. Their counter theory says that the design of architecture is not merely a construction of tokens within a logic system but rather a constant reflective conversation. That is - we approach design with a pre-understanding, a vague projection of the completed product as the parts are assembled. This projection is then re-projected in a dialectical fashion to refine and redefine the parts.

Irrespective of which of these theories you subscribe to, it can generally be agreed that design works because it is embedded in a human situation or it is an attribute of our relationship with our environment, culture, and personal memories. Because of this, the creator of architecture, like the author of a linguistic work must deal with the

disconnection of his/her mental intention (during design) from the verbal meaning of the built work (after design). Marshall McLuhan [2] derives from Gestalt psychology of 'figure-ground relationships' - to fully grasp the effect of an artifact, one must examine the artifact as the figure (medium) and the socio-cultural context as ground (context). What he means is that not only must the figure and ground be evaluated together, but also as a critique to one another. In particular this is important since neither is completely intelligible without the other.

Architecture, like the written text is addressed to an unknown reader and in fact, to everyone who can read it. On one hand, this semantic autonomy opens up the range of potential readers, and so creates the architecture's audience. On the other hand, it is the response of the audience that gives the architecture its context, and significance [4]. Thus, the narrative of architecture from the architect's perspective may be completely different from the user's perspective. Moreover, each participant in this conversation may have a different narrative... which is why a tourist has a different relationship with Crown Hall than an architecture student who studies in its hallowed halls and breathes its air day in and day out. And both are acceptable for architecture.

An effective 'place' design is one that can cross over the limits enforced by abstract experience into the semiotic constructs required for linguistic expression. As sensuous beings, we experience 'place' as self, yet our sensibility of this experience is defined by our relationship with the other. This scope for narrative is what defines our experiences. A narrative establishes the threshold through which we can frame references to our experience and in turn transfer these references to others. This is why stories we tell are seldom about buildings, or cities, or walls, or doors, or furniture, but always about our lives around them.

For the Spring 2008 issue of ARCC Journal, we were interested in work that evaluates new methods and manners in the semantic appropriation of architecture. We invited authors to submit papers which explored expression 
and communication in its various forms in the design and/or critique of architecture. Authors could submit empirical research that dealt with the design of architecture (and/or its elements) or speculative research that looked at theoretical frameworks for the design of architecture (and/or its elements). Themes included (but did not limit to) study of historic precedents and theoretical constructs in the relationship of language and architecture; the use of semantic expression in the teaching of architecture; new semantic appropriations of architecture as enabled by new technologies; sustainability as a form of expression in architecture/design; as well as the evolution and extinction of architectural entities and philosophies in evolving sociocultural contexts.

This issue of the ARCC journal consists of six papers, three peer-reviewed and three invited, all of which explore different perspectives of architecture as a communicative medium.

The first of the first of the peer reviewed papers, "On dependencies between architecture and media: considering the remote work" by Christenson, runs through a careful review of the communicative aspects of architecture, bringing forth the importance of mediating artifacts in the representation and discussion of architecture. He proposes the use of two unique frameworks in the analysis of mediated artifacts (in this case, photographs) and presents empirical studies to evaluate these hypotheses. Downing et al. present the ideas of embodiment and its significance to the design in their paper "An Embodied Architecture". Using the framework of interactions at the edge of dual/multiple systems, the authors present notions of edge and significance of form as critical to the making and perception of architecture. Teal, in his paper, "Intuition as Design Dialogue: Discovering a Language beyond Words" presents the notion of intuition as critical to the construction of design knowledge. He puts forth intuition and intellect as concepts which enable a person to develop intrinsic understanding of a certain subject and conclude with work from design studios to illustrate different ways in which intuition is supported in a pedagogical environment.

Anders' paper, "The Planetary Collegium: Master Plan for a Distributed Mixed Reality Campus", which begins the invited segment, presents a new method of collaboration within physical and virtual spaces using Mixed Reality. His paper also presents the concepts of cybrids - a hybrid of physical programming with virtual programming through the connections of cybernetics. Haddad explores the notion of identity in his paper - "Learning from Beirut: From Modernism to Contemporary Architecture". Through the canvas of Lebanon's modern and postmodern architecture, the paper explores social and political impetus that defined Lebanon's search for cultural identity. And finally Monson's paper, "Semiotics of making: beginnings of a theoretical frame" uses Elaine Scarry's text, The Body in Pain, to explore the symbolic nature of making, and its relationship to understanding architecture and the design of architecture.

\section{References}

1. Auburn, T. and Barnes, R. Producing place: A neo-Schutzian perspective on the 'psychology of place'. Journal of Environmental Psychology, 26 (1). 38-50.

2. McLuhan, M. and McLuhan, E. Laws of Media: The New Science. University of Toronto Press, 1988.

3. Mitchell, W.J. The Logic of Architecture: Design, Computation, and Cognition. MIT Press Cambridge, MA, USA, 1990.

4. Ricoeur, P. Interpretation Theory: Discourse and the Surplus of Meaning. Texas Christian Univ Pr, 1976.

5. Snodgrass, A.B. and Coyne, R.D. Is Designing Hermeneutical? Architectural Theory Review. Journal of the Department of Architecture, The University of Sydney, 1 (1). 65-97 